In Crescendo, 2019; 10(1): 161-173

Fecha de recepción: 19 de enero del 2019

Fecha de aceptación: 25 de marzo del 2019

\title{
MONITOREO BASADO EN DASHBOARD Y SU EFECTO EN EL CUMPLIMIENTO DE LOS ESTÁNDARES DE ACREDITACIÓN
}

\author{
MONITORING BASED ON DASHBOARD AND ITS EFFECT \\ ON COMPLIANCE WITH THE ACCREDITATION \\ STANDARDS
}

\author{
Miguel A. Valles', Luis M. Hidalgo', \\ John C. Santa-María ${ }^{3}$
}

\section{RESUMEN}

En 2018, la Universidad Nacional de San Martín - T (UNSM-T) realizó la autoevaluación de sus programas de estudio, para lo cual usó una herramienta basada en Excel del Sistema Nacional de Evaluación, Acreditación y Certificación de la Calidad Educativa (SINEACE), el cual cumple estructuralmente con la matriz de evaluación, pero carece de funciones que resultan indispensables para que las Escuelas Profesionales puedan manejar adecuadamente sus recursos generando incertidumbre al realizar el monitoreo. En ese sentido el objetivo fue implantar un sistema de monitoreo basado en dashboard para mejorar el seguimiento y evaluación del cumplimiento de los estándares de acreditación de los programas de estudio de la UNSM-T. Para ello se realizó un estudio descriptivo comparativo, aplicado, transversal a 60 miembros de los comités de acreditación a los que se aplicó un instrumento para medir su opinión respecto al monitoreo del cumplimiento de los estándares de acreditación en la UNSM-

1 Ing. de Sistemas, Director (e) Unidad de Investigación. Facultad de Ingeniería de Sistemas e Informática - Universidad Nacional de San Martín-Tarapoto, Perú.mavalles@unsm.edu.pe

2 Bach. de Sistemas, Tesista. Facultad de Ingeniería de Sistemas e Informática - Universidad Nacional de San Martín - Tarapoto, Perú.lmhidalgo@unsm.edu.pe.

3 Ing. de Sistemas, Director (e) Escuela Profesional de Sistemas e Informática - Universidad Nacional deSan Martín - Tarapoto, Perú. jsantamaria@unsm.edu.pe. 
T, antes y después de la propuesta. Los resultados nos indican que 91.7\% opina que la propuesta es buena en comparación a la propuesta en Excel. Se concluye que la propuesta permite realizar un adecuado seguimiento y evaluación del cumplimiento de los estándares de acreditación de los programas de estudio de la UNSM-T.

PALABRAS ClAVE: Acreditación, Evaluación, Monitoreo, Seguimiento, Tablero.

\section{ABSTRACT}

In 2018, the National University of San Martín - T (UNSM-T) carried out the self-evaluation of its study programs, for which it used an Excel-based tool of the National System of Evaluation, Accreditation and Certification of Educational Quality (SINEACE), which structurally complies with the evaluation matrix, but lacks functions that are indispensable for the Professional Schools to adequately manage their resources, generating uncertainty when performing the monitoring. In this sense, the objective was to implement a monitoring system based on a dashboard to improve the monitoring and evaluation of compliance with the accreditation standards of the UNSM-T study programs. To this end, a comparative descriptive study was carried out, applied to 60 members of the accreditation committees to which an instrument was applied to measure their opinion regarding the monitoring of compliance with the accreditation standards in the UNSM-T, before and after of the proposal. The results indicate that $91.7 \%$ think that the proposal is good compared to the one proposed in Excel. It is concluded that the proposal allows an adequate follow-up and evaluation of compliance with the accreditation standards of the UNSM-T study programs.

KEY WORDS: Accreditation, Evaluation, Monitoring, Monitoring, Board.

\section{INTRODUCCIÓN}

La Universidad Nacional de San Martín - Tarapoto, como parte del proceso de mejora continua, paralelo al proceso de licenciamiento, ha venido trabajando articuladamente con los diferentes programas académicos, a fin de lograr su acreditación para así garantizar que las competencias profesionales de sus egresados sean adecuadas y reflejen una formación educativa de calidadSINEACE, (2018), para ello ha seguido las recomendaciones formuladas por el Sistema Nacional de Evaluación, Acreditación y Certificación de la Calidad Educativa, algo que en Chile, Colombia y otros países de la región tiene un mayor nivel de avance como nos lo explica Jiménez (2014), en su artículo "Cuadro de Mando Integral para la implementación curricular por competencias para una Institución Universitaria”. 
En ese sentido, para "lograr la calidad en las carreras universitarias supone un esfuerzo continuo por cumplir exigencias en docencia, investigación, extensión universitaria y vinculación social; por ejemplo, desempeño profesional de los graduados, producción científica de los docentes, calidad de procesos pedagógicos y eficiencia en el manejo de recursos" Acosta \& Acosta, (2016), se ha llevado a cabo un proceso de autoevaluación o evaluación interna con los comités de calidad de los programas académicos, lo cual implicaba incorporar en el proceso, una herramienta informática basada en Excel proporcionada por SINEACE.

Esta herramienta, si bien cumple estructuralmente con la Matriz de Evaluación para la Acreditación de los programas de estudio y es medianamente prácti$\mathrm{ca}$, carece de funcionalidades integradoras que resultan importantes para que las escuelas profesionales, la Oficina de Acreditación y el Rectorado, controlen, monitoreen, den un adecuado seguimiento, evaluación y retroalimentación al logro de los estándares de acreditación que al ser difíciles de realizar coincidimos con Villacís, (2017) al afirmar que en su web "gerente que no da seguimiento, causa muchos problemas" que "el no dar seguimiento hasta que los resultados de un proyecto se perfeccionen, o el no dar seguimiento a que las actividades cotidianas no se desvíen de su cauce, origina problemas de comunicación", de manera que no se pueden tomar adecuadas decisiones para la redistribución de plazos, recursos o entregables en función a los avances logrados por los programa de estudio como lo indica además la Organización de Estados Iberoamericanos, (2015) que en su "teoría de la planificación del desarrollo define el seguimiento o monitoreo como un ejercicio destinado a identificar de manera sistemática la calidad del desempeño de un sistema, subsistema o proceso a efecto de introducir los ajustes o cambios pertinentes y oportunos para el logro de sus resultados y efectos en el entorno".

La evaluación, seguimiento y monitoreo del cumplimiento de los estándares de acreditación implica proporcionar una visión estratégica del proceso, en el que se conozcan y deleguen responsabilidades, recursos, plazos y metas, de tal manera que se asegure la calidad educativa de los programas de estudio de la UNSM-T, como así lo indicaNuñez Martín, (2018) al afirmar que "la mentalidad estratégica implica ir más allá de la gestión del día al día, establecer metas y objetivos, determinar y planificar cada uno de los retos y una vez alcanzados, evaluarlos y medirlos para comprobar si lo proyectado coincide con las expectativas", en busca del mismo, nos preguntamos si es posible mejorar el seguimiento y evaluación del cumplimiento de los estándares de acreditación de los programas de estudio de la 
UNSM-T, al incorporar en el proceso un sistema de monitoreo basado en dashboard para que los responsables (a nivel estratégico, táctico y operativo), conozcan cuáles son las dimensiones, factores y estándares cuyo cumplimiento están aportando cuantitativamente al proceso de acreditación del programa académico y su agregación a nivel institucional a fin de evaluar las acciones correctivas si fuera el caso o retroalimentarse a partir de las experiencias exitosas del proceso.

El objetivo fue proporcionar una herramienta basada en dashboard para mejorar el seguimiento y evaluación del cumplimiento de los estándares de acreditación de los programas de estudio de la UNSM-T, implementando un sistema de monitoreo del cumplimiento de los estándares de acreditación basado en dashboard ya que según Elósegui, (2014), "un dashboard nos permite hacer un seguimiento del grado de cumplimiento de los objetivos de negocio", luego ejecutar un piloto de implantación del sistema en los programas muestra del estudio y evaluar el impacto de la herramienta de monitoreo sobre el seguimiento y evaluación de los estándares de acreditación.

Los resultados del estudio nos indican que el 91,7\% de los encuestados opinan que la propuesta para monitorear el proceso de evaluación y seguimiento del logro de los estándares de acreditación es buena en comparación de la propuesta basada en una plantilla en Excel del SINEACE, además se ha logrado implantar el sistema en la siguiente dirección: http://acreditacion.unsm.edu.pe/ acreditacion.

\section{MÉTODOS}

El universo del estudio han sido los integrantes de los comités de calidad de los programas de estudio de la UNSM-T que en total suman 105, con una muestra de 60 personas, en un estudio descriptivo comparativo, de tipo aplicado, de corte transversal en el que se aplicó un instrumento para medir la apreciación que tienen respecto al seguimiento y evaluación del cumplimiento de los estándares de acreditación de los programas de estudio de la UNSM-T en un primer momento con la herramienta Excel diseñada y proporcionada por SINEACE y luego con el uso del sistema de monitoreo basado en dashboard de nuestra propuesta.

El proyecto inició con el levantamiento de información en coordinación con la Oficina de Acreditación y Calidad Académica, así como también con los comités de calidad de los programas de estudio de la UNSM - T para conocer la problemática respecto a la acreditación, concluyendo que el sistema de monitoreo diseñado y 
proporcionado por SINEACE cumple con la matriz SINEACE, (2016) pero no brinda las funcionalidades necesarias para realizar un correcto seguimiento y evaluación del cumplimiento de los estándares de acreditación, entre ellos la carencia de indicadores claves de desempeño genera retraso en la toma decisiones por parte de la oficina, que actúa de manera sesgada en este proceso.

Además se presenta la deficiente comunicación entre la Oficina de Acreditación y Calidad Académica de la UNSM - T y dichos comités y para Villacís, (2017) "dar seguimiento efectivo a tus clientes, requiere una revisión de tus habilidades de comunicación y supervisión”. La herramienta basada en Excel adolece gravemente de un esquema de centralización de la información y todo lo contrario es un esquema repartido sin comunicación, en consecuencia la retroalimentación que debería darse entre estos dos actores en el proceso de acreditación se lleva a cabo de manera descoordinada y lenta, siendo obligatorio para la universidad proporcionar herramientas que permitan un mejor trabajo a nivel estratégico como lo indica (Luis Almuiñas Rivero \& Galarza López, 2012) al finar que "ello obliga a las organizaciones, incluyendo las universidades, a reaccionar ante los riesgos y oportunidades con una nueva forma de sustentar su gestión. Uno de los enfoques, que ha ganado auge en las universidades latinoamericanas es la dirección estratégica, dentro de la cual se le está prestando especial atención a la planificación estratégica, como herramienta para orientar el rumbo institucional".

Luego, se diseñó el nuevo sistema, tomando lo básico del sistema anterior como punto de partida, agregando funcionalidades, las que mejoran la manera de realizar el monitoreo, ya que como lo indica Martínez, (2017), "el dashboard es una herramienta que permite hacer un seguimiento y evaluación de situaciones concretas, además de realizar un diagnóstico basado en una serie de indicadores y evidencias, a través de herramientas visuales"

Fue necesario entonces diseñar un flujo de procesos que permita mejorar la comunicación, permitiendo una adecuada retroalimentación, garantizando además que las observaciones a la documentación y medios de verificación se canalicen centralizadamente a través del sistema propuesto, tratando de asegurar que no ocurra lo que precisa (Becerra, Andrade, \& Díaz, 2017) cuando dice que "el modo en que las universidades han adoptado un enfoque centrado en la gestión de la calidad para lograr una mayor eficiencia y eficacia en la dirección de los procesos universitarios, y asegurar su mejora continua, es uno de los problemas principales de la gestión universitaria moderna". 
Es así que el flujograma de la propuesta quedó de la siguiente manera:

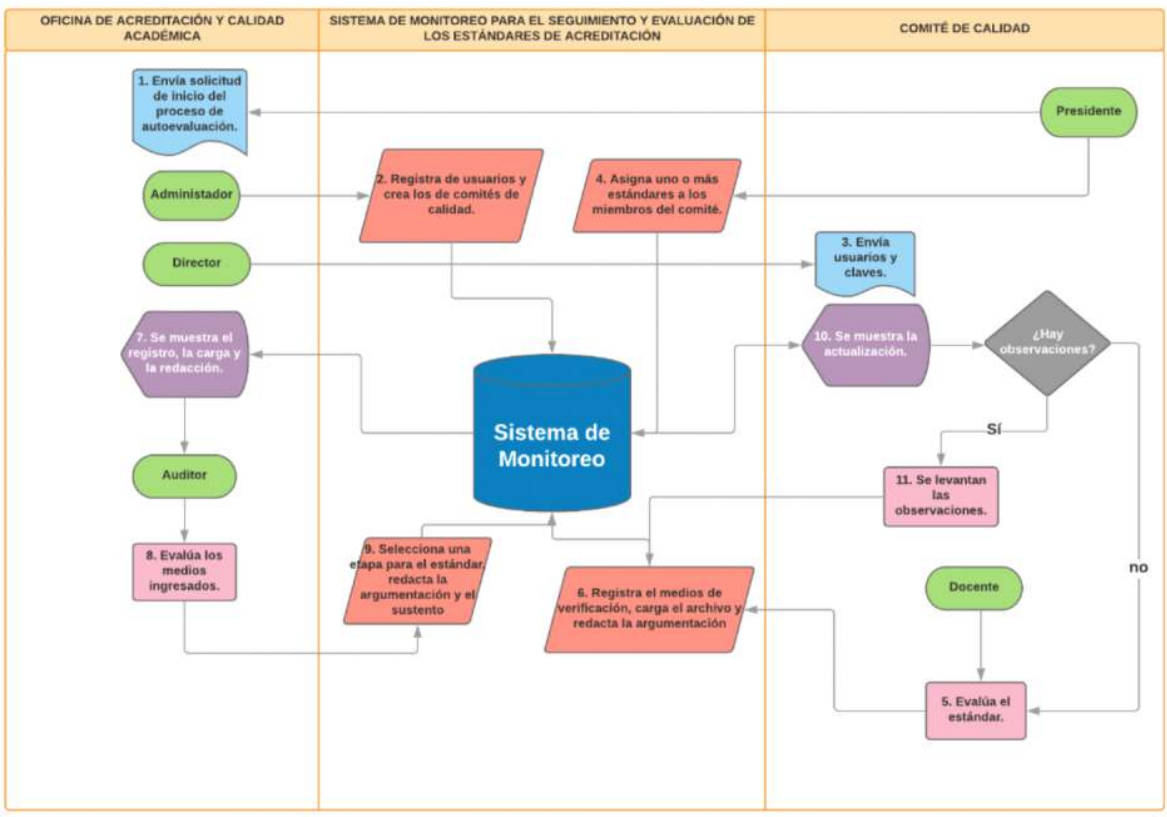

Figura 1. Flujograma del sistema de monitoreo basado en Dashboard.

Las funcionalidades de la propuesta, como se menciona antes se caracterizan por estar centralizadas en una base de datos única, además el sistema se ha construido bajo plataforma web con diseños responsivos por lo que se adecúan fácilmente para su uso en smartphones, siendo las nuevas funcionalidades: Parametrización del sistema.

Asimismo, Permite que la Oficina de Acreditación y Calidad Académica configure la matriz de evaluación a usarse en función a la programa de estudios que puede ser delICACIT, (2018) oSINEACE, (2018), además de realizar toda la parametrización necesaria para crear programas académicos, comités de calidad, plazos para la presentación de medios de verificación y demás parámetros del sistema. Asignar estándar. Permite al presidente del comité de calidad asignar estándares de acreditación a cada miembro como responsable del cumplimiento de ese estándar. Estado del estándar. Permite conocer el estado real de los estándares. Pueden ser: Pendiente, No Logrado y Logrado. Generar reportes. Los mismos que son solicitados por la Oficina de Acreditación y Calidad Académica para la autoevaluación. 
Nivel de avance. Acceso al tablero de comando o Dashboard, el cual proporciona datos porcentuales del cumplimiento de los estándares de acreditación. Evaluación del estándar. Es la funcionalidad más importante de la propuesta en la cual se da el flujo principal de todo el proceso. Comparación del avance. Esta funcionalidad es exclusiva del miembro auditor de la Oficina de Acreditación y Calidad Académica de la UNSM - T, permite visualizar el nivel avance de manera porcentual, mediante filtros: Formato SINEACE, (2016) o del Instituto de Calidad y Acreditación de Programas de Computación, Ingeniería y Tecnología en ingeniería - ICACIT, (2018), programa de estudio, dimensión, factor y estándar.

Para la construcción del software, se ha seguido estrictamente el diseño, se tuvo una primera versión la cual se presentó a los interesados, esto nos ha proporcionado retroalimentación para mejorar la propuesta. Se realizaron las actualizaciones correspondientes a nivel de software para mejorar la propuesta, logrando de esta manera que el sistema de monitoreo basado en Dashboard satisfaga las necesidades tanto de los miembros de la Oficina de Acreditación y Calidad Académica, así como también del comité de calidad de los programas de estudio.

Una vez que la propuesta de monitoreo basado en Dashboard tuvo el visto bueno, se procedió a la puesta en producción del sistema, comenzando de esta manera el periodo de prueba en el cual se ingresan datos a la plataforma durante un tiempo determinado y se observa su comportamiento.

A su vez se hizo la presentación oficial del sistema durante una reunión que sirvió a la vez como capacitación que de acuerdo a la Federación Internacional de Sociedades de la Cruz y de la Media Luna (2011) "consiste en cursos y talleres sobre la planificación de las actividades de seguimiento y evaluación; la recopilación, la gestión y el análisis de datos; y la elaboración de informes, entre otras tareas", en el que se aplicó un pre-test para conocer la opinión que tiene cada uno de los actores en el proceso de acreditación con respecto al sistema de monitoreo propuesto por el SINEACE, una vez concluido el pre-test, se expuso la propuesta de la investigación, explicando el nuevo flujograma de procesos, las funcionalidades del sistema y la propuesta propiamente dicha, dando a conocer los beneficios que trae el uso del software en el proceso de acreditación de programas de estudio.

Luego de la presentación de la propuesta, se aplicó el post-test, con un instrumento que contaba con las mismas preguntas del pre-test, pero respecto a la propuesta presentada, de esta manera se logró recolectar datos cuantitativos de ambos escenarios, se hizo el procesamiento de la información y se determinó la 
validez de la hipótesis. El instrumento diseñado es una "Encuesta para medir la apreciación sobre el seguimiento y evaluación del cumplimiento de los estándares de acreditación de los programas de estudio de la Universidad Nacional de San Martín - Tarapoto". La propuesta, se centra en el sistema de monitoreo basado en dashboard como eje de todo el proceso de seguimiento y evaluación del cumplimiento de estándares de acreditación de los programas de estudio, esto permite tener datos reales y centralizados, accesibles desde cualquier plataforma, pero sobre todo información confiable. Con los datos, se generan reportes de suma importancia, así mismo el dashboard brinda una visión general del proceso de autoevaluación hasta una más específica, facilitando el seguimiento y evaluación por parte del auditor interno y del presidente del comité de calidad, para la toma de decisiones siguiendo indicadores clave de desempeño.

\section{RESULTADOS}

Para obtener los resultados del estudio se ha trabajado durante 5 meses, obteniéndose lo siguiente:

Implementar un sistema de monitoreo del cumplimiento de los estándares de acreditación basado en Dashboard. Se ha construido el sistema aplicando adecuadamente la metodología para el diseño de un Dashboard de Martínez, (2017), teniendo como principales interesados a la Oficina de Acreditación y Calidad Académica y los Comités de Calidad de las Escuelas Profesionales. El sistema se ha dejado instalado, configurado y en funcionamiento en la siguiente dirección web: http://acreditacion.unsm.edu.pe/acreditacion

Iniciar un piloto de implantación del sistema en los programas muestra del estudio. Se ha iniciado el piloto de implantación del sistema de monitoreo basado en Dashboard con la participación de las escuelas de Obstetricia, Enfermería, Ingeniería de Sistemas, Agroindustrias y Agronomía, sin embargo, también participaron en la presentación del sistema las escuelas de Arquitectura, Economía, Contabilidad, Turismo, Idiomas.

Evaluar el impacto de la herramienta de monitoreo sobre el seguimiento y evaluación de los estándares de acreditación. A continuación se muestra resultados comparativos sobre la opinión que tienen los miembros del comité de acreditación sobre el seguimiento y evaluación del cumplimiento de los estándares de acreditación, según la propuesta deSINEACE, (2018) y según la propuesta de la Investigación. 
Tabla 1

OPINIÓN SOBRE EL PROCESO DE SEGUIMIENTO Y EVALUACIÓN SEGÚN HERRAMIENTA

\begin{tabular}{lcc}
\hline Opinión & \multicolumn{2}{c}{ Herramienta de Monitoreo } \\
& Dashboard & Sineace \\
\hline Malo & $0,0 \%$ & $70,0 \%$ \\
Regular & $8,3 \%$ & $30,0 \%$ \\
Bueno & $91,7 \%$ & $0,0 \%$ \\
\hline
\end{tabular}

Fuente: Datos obtenidos del cuestionario aplicado a los Docentes Integrantes de los Comité de Calidad de la UNSM-T

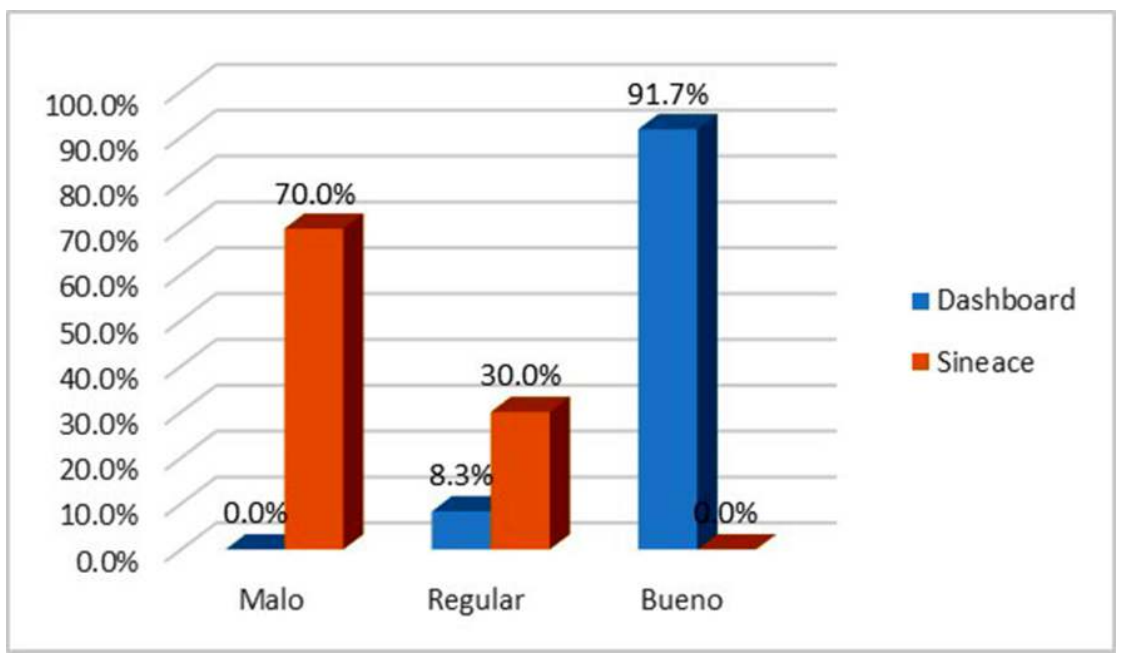

Figura 1. Apreciación sobre el seguimiento y evaluación del cumplimiento de los estándares de acreditación según propuesta.

Interpretación. En la tabla y figura 1, según el resultado se evidencia que el $70 \%$ de los docentes opinan que el proceso actual es Malo, un 30\% opinan que es regular y el 0\% opinan que es Bueno; así mismo la opinión sobre la propuesta basada en Dashboard es que el 0\% opinan que es mala, un 8.3\% opinan que es regular y un $91,7 \%$ opinan que es buena. 


\section{DISCUSIÓN}

Los resultados son lo que se esperaba y no distan mucho de lo encontrado por Arias, (2015), ya que él nos indica que "los resultados obtenidos durante la ejecución del proyecto estuvieron de acuerdo a lo esperado ya que por medio del tablero de control y la agrupación de las principales variables que impactan a los proyectos como los riesgos, problemas y tiempos se tiene una visión global sobre el portafolio de los proyectos para la toma de decisiones de acuerdo a la información histórica obtenida" y también por Mahecha (2017), respecto a que un Sistema de Monitoreo Basado en Dashboard mejora el seguimiento y evaluación de los indicadores claves de desempeño identificados en un proceso determinado y que es respaldado por Valles \& Rengifo (2018) quienes, producto de la incorporación del componente de tecnología de información y comunicaciones en los procesos, han logrado optimizar la gestión de los indicadores de la investigación en su estudio.

Siguiendo con el tema, de acuerdo a Santa Cruz (2018), la implementación de una solución de inteligencia de negocios dentro del ambiente operativo de las empresas del Grupo Palmas fue un logro significativo que definitivamente marcó una brecha importante entre la situación en la que se encontraba trabajando antes y después de la adquisición de la solución propuesta, en ese sentido, nuestra propuesta también coincide con los resultados del estudio de Santa Cruz (2018), ya que si bien es cierto la solución no se ha llegado a usar de modo masivo como lo hubiéramos deseado, sí existe una opinión positiva (cercana al 100\%) de la mejora en el proceso de seguimiento y evaluación del cumplimiento de los estándares de acreditación de los programas de la UNSM-T.

Por su parte nuestro estudio también coincide con los resultados de Alfaro y Paucar, (2016), quienes nos indican que "lograron acopiar información de tendencias e indicadores de grandes cantidades de datos de los incidentes reportados por los usuarios a la Mesa de Ayuda, para el análisis rápido y oportuno se generan reportes de manera automática con gráficos estadísticos y Dashboards", en nuestro caso también nuestro sistema posee como funcionalidad principal un Dashboard que presenta gráficos estadísticos a modo de tablero en el que se puede ver de manera automática el incremento porcentual del logro de los indicadores de acreditación de los diferentes programas, proporcionando una perspectiva gerencial y táctica para gestionar mejor los procesos operativos de la acreditación. 


\section{CONCLUSIONES}

Siguiendo la metodología propuesta por Martínez (2017) se ha construido el sistema de Monitoreo del Cumplimiento de los Estándares de Acreditación basado en Dashboard para la Oficina de Acreditación y Calidad Académica, proceso que ha sido dirigido con la participación en todas las fases del proceso del personal especialista en acreditación con el que cuenta la oficina y los usuarios finales que vendrían a ser los integrantes de los comités de calidad de las escuelas profesionales de la Universidad, garantizando así que la propuesta tenga un alto grado de participación de tal manera que aseguremos la aceptación al momento de la masificación durante la fase de implantación a mayor escala y mediante normativas y procedimientos rectores aprobados a llevarse a cabo en el 2019.

Se ha iniciado el piloto de implantación del sistema con la presentación del mismo en reuniones realizadas con los comités de acreditación de las escuelas profesionales de Enfermería, Obstetricia, Ingeniería de Sistemas, Ingeniería Agrónoma, Medicina Veterinaria y Turismo. Además, en coordinación con la oficina de Acreditación y Calidad Académica se ha realizado una presentación del piloto a todas las carreras profesionales en la sala de reuniones de la misma oficina, en la que participaron los comités de calidad de las escuelas profesionales de la UNSM-T.

Finalmente, mediante la aplicación de un instrumento (encuesta) se ha evaluado la opinión que tienen los comités de acreditación sobre el impacto que tendrá la propuesta de Monitoreo basada en Dashboard y se ha podido determinar que el $91.7 \%$ de los docentes integrantes de los comités de calidad consideran que será bueno, en comparación con el 70\% que opinan que la propuesta del SINEACE basada en Excel es mala, para realizar un adecuado seguimiento y evaluación del cumplimiento de los estándares de acreditación de programas de estudio de la Universidad Nacional de San Martín - Tarapoto.

Teniendo esto en cuenta los resultados, se recomienda formular normas y directivas para asegurar una implantación exitosa de la propuesta, además se sugiere desagregar los comités de acreditación en "avanzados" y "en proceso", por último, aprovechar la alta aceptación de la propuesta para socializar, concientizar y masificar la adaptación de la misma. 


\section{REFERENCIAS BIBLIOGRÁFICAS}

Acosta, B., \& Acosta, M. (2016). Modelos de evaluación para acreditación de carreras. Análisis de su composición y una propuesta para las carreras de Ecuador. Revista Mexicana de Investigación Educativa, 21(71), 1249-1274. Retrieved from http://www.scielo.org.mx/ scielo.php?pid $=$ S1405-66662016000401249\&script $=$ sci_arttext\&tlng $=$ en

Alfaro, L., \& Paucar, D. (2016). Construcción de un Datamart que apoye en la toma de decisiones de la gestión de incidencias en una mesa de ayuda: caso Consorcio Peruano de Empresas. Repositorio de Tesis - UNMSM. Universidad Nacional Mayor de San Marcos. Retrieved from http://cybertesis.unmsm.edu.pe/handle/cybertesis/5209

Arias, C. (2015). Implementación de un Dashboard para el seguimiento de portafolio de proyectos. Universidad Pontificia Bolivariana. Retrieved from https://repository.upb.edu.co/ bitstream/handle/20.500.11912/2995/Informe Final Andres Arias.pdf?sequence=1

Becerra, A., Andrade, A., \& Díaz, L. (2017). Sistema de Información Estratégica para la Gestión Universitaria en la Universidad de Otavalo, Ecuador. Formación Universitaria, 10(2), 103. 112. https://doi.org/10.4067/S0718-50062017000200011

Elósegui, T. (2014). ¿Qué es un dashboard y para qué sirve? Retrieved December 12, 2018, from https://tristanelosegui.com/2014/10/27/que-es-y-para-que-sirve-un-dashboard/

Federación Internacional de Sociedades de la Cruz Roja y de la Media Luna Roja. (2011). Guía para el seguimiento y la evaluación de proyectos y programas, 162. Retrieved from http://www.ifrc.org/Global/Publications/monitoring/1220500-Monitoring-and-Evaluationguide-SP.pdf

ICACIT. (2018). Criterios de Acreditación de Programas de Ingeniería. Lima. Retrieved from www.icacit.org.pe

Jiménez, J. (2014). Cuadro de Mando Integral para la Implementación Curricular por Competencias para una Institución Universitaria. Revista de Estudios y Experiencias En Educación, 13(26), 169-182. Retrieved from http://www.redalyc.org/articulo.oa?id= $243132847010 \% 5 \mathrm{CnC}$ mo

Luis Almuiñas Rivero, J., \& Galarza López, J. (2012). El proceso de planificación estratégica en las universidades: Desencuentros y retos para el mejoramiento de su calidad. Revista Gestao Universitaria Na America Latina, 5(2), 72-97. https://doi.org/10.5007/1983 $4535.2012 \mathrm{v} 5 \mathrm{n} 2 \mathrm{p} 72$

Mahecha, H. (2017). Implementación de una herramienta 'Dashboard' para el control y gestión de procesos automatizados en ColPensiones. Universidad Nacional Abierta y A Distancia. Retrieved from https://stadium.unad.edu.co/preview/UNAD.php?url=/bitstream/10596/ 11928/3/1072747124.pdf

Martínez, D. (2017). Metodología para el diseño de un Dashboard orientado hacia el registro de evidencias en el proceso de evaluaciones institucionales. Puyo.

Nuñez Martín, A. (2018). La visión estratégica del CEO. Retrieved January 8, 2019, from https://mba.americaeconomia.com/articulos/reportajes/la-vision-estrategica-del-ceo 
Organización de Estados Iberoamericanos. (2015). Monitoreo e indicadores. Guatemala: Instituto para el desarrollo y la innovación educativa. Retrieved from http://www.oei.es/idie/ monitoreoeindicadores.pdf

Santa Cruz, W. (2018). Business Intelligence aplicada al monitoreo de la producción en las empresas agrícolas del Grupo Palmas 2015. Universidad Nacional de San Martín. Retrieved from http://repositorio.unsm.edu.pe/bitstream/handle/UNSM/2912/SISTEMAS - Winser Santa Cruz Lopez.pdf? sequence $=1$ \& is Allowed $=y$

SINEACE. (2016). Modelo de Acreditación para Programas de Estudios de Educación Superior Universitaria. Retrieved December 16, 2018, from https:/www.sineace.gob.pe/wpcontent/uploads/2014/08/Anexo-1-nuevo-modelo-programas-Resolucion-175.pdf

SINEACE. (2018). Acreditación. Sistema Nacional de Evaluación. Retrieved December 8, 2018, from https://www.sineace.gob.pe/acreditacion/

Valles Coral, M. A., \& Rengifo Arias, M. A. (2018). Estrategia de TI para el control de indicadores de gestión en la investigación en la UNSM-T, 2017. Tarapoto: Universidad Nacional de San Martín - Tarapoto. Retrieved from http://repositorio.unsm.edu.pe/bitstream/handle/ UNSM/2913/INVESTIGACION - Miguel Angel Valles Coral \%26 Miguel Angel Rengifo Arias.pdf? sequence $=1$ \&isAllowed $=\mathrm{y}$

Villacís, P. (2017). Gerente que no da seguimiento, causa muchos problemas. Retrieved December 12, 2018, from https://www.salesup.com/crm-online/cc-dar-seguimiento-gerentes.shtml 